\section{Desenvolvimento de um Questionário Quantitativo de Freqüência Alimentar (QQFA) para gestantes usuárias de unidades básicas de saúde de Ribeirão Preto, São Paulo, Brasil}

\author{
Development of a quantitative food frequency \\ questionnaire for pregnant women attending \\ primary care in Ribeirão Preto, São Paulo \\ State, Brazil
}

\author{
1 Faculdade de Medicina de \\ Ribeirão Preto, Universidade \\ de São Paulo, Ribeirão Preto, \\ Brasil. \\ 2 Faculdade de Medicina \\ de Botucatu, Universidade \\ Estadual Paulista Júlio de \\ Mesquita Filho, Botucatu, \\ Brasil. \\ Correspondência \\ D. S. Sartorelli \\ Departamento de Medicina \\ Social, Faculdade de \\ Medicina de Ribeirão Preto, \\ Universidade de São Paulo. \\ Av. Bandeirantes 3900 , \\ Ribeirão Preto, SP \\ 14049-900, Brasil. \\ daniss@fmrp.usp.br
}

\begin{abstract}
The aim of this study was to design a food frequency questionnaire for pregnant women attending primary care under the Unified National Health System in Ribeirão Preto, São Paulo State, Brazil. A 24-hour food recall was obtained from 150 pregnant women, 50 in each trimester of pregnancy (age 18-35 years). A second food recall was obtained from 90 women, 30 in each trimester of pregnancy, from 7 to 15 days after the first dietary assessment. An 85-item questionnaire was developed with stepwise multiple linear regression models, based on the contribution of each food to inter-individual variation of energy and 28 target nutrients, which accounted for 74.2 to 98.8\% of the selected nutrients. Portion sizes were determined according to percentiles 25, 50, 75, and 100 for each food item intake. To our knowledge, this is the first food frequency questionnaire designed for Brazilian pregnant women.
\end{abstract}

Food Intake; Pregnant Women; Nutritional Epidemiology
Thaís de Oliveira 1

Fabíola Darcie Marquitti 1

Maria Antonieta de Barros Leite Carvalhaes 2 Daniela Saes Sartorelli 1

\section{Introdução}

A gestação consiste num período caracterizado por intensas modificações fisiológicas, metabólicas e endócrinas, que alteram as necessidades nutricionais e a ingestão alimentar 1 . O estado nutricional materno e o ganho de peso gestacional influenciam o risco de morbimortalidade materna, fetal e a saúde do futuro adulto. O déficit de peso pré-gestacional e o ganho de peso materno insuficiente vêm sendo associados ao baixo peso ao nascer 2 . Por sua vez, o ganho de peso materno excessivo constitui um importante fator de risco para macrossomia 3,4 , diabetes gestacional 4,5, pré-eclâmpsia 4,5 e complicações no parto 5,6, comprometendo a saúde do binômio mãe-filho.

A ingestão alimentar materna habitual é uma das determinantes do ganho de peso na gestação, o que está associado direta ou indiretamente ao desenvolvimento de complicações durante a gestação 7,8 .

Um estudo de coorte, conduzido nos Estados Unidos com 1.718 gestantes, verificou que baixos teores de cálcio, ácidos graxos poliinsaturados e trans, magnésio, folato e vitaminas C, D e E estavam associados ao maior risco de pré-eclâmpsia e hipertensão gestacional 9. Outra coorte realizada nos Estados Unidos, com amostra de 832 gestantes, constatou o papel protetor do folato na dieta contra o desenvolvimento de anemia megaloblástica, prematuridade e baixo peso ao 
nascer 10. Clapp et al. 11 investigaram a relação entre carboidratos de alto índice glicêmico na dieta materna e o excesso de peso pré-gestacional para o risco de diabetes gestacional e macrossomia fetal. No outro extremo, a restrição severa de alimentos com elevado índice glicêmico em mulheres eutróficas e/ou baixo peso pré-gestacional pode predispor ao baixo peso ao nascer 12 . Tais estudos ressaltam a importância da investigação da relação entre a ingestão alimentar habitual de gestantes e desfechos maternos e infantis, relevante para a tomada de decisões no âmbito da atenção pré-natal e também para definição de políticas de promoção da saúde desse grupo populacional.

A investigação da associação entre dieta e ocorrência de doenças em estudos de epidemiologia nutricional requer o emprego de métodos de avaliação da ingestão alimentar precisos, acurados e viáveis ${ }^{13}$. Entretanto, a obtenção de dados precisos é uma tarefa difícil, uma vez que não há um padrão ouro para avaliação da ingestão de alimentos e nutrientes, e os métodos disponíveis estão sujeitos a variações e erros de medida ${ }^{13}$. O Questionário Quantitativo de Freqüência Alimen$\operatorname{tar}$ (QQFA) é amplamente empregado em estudos epidemiológicos, pois avalia a ingestão alimentar habitual em período retrospectivo de tempo, é de baixo custo e permite a categorização dos indivíduos em níveis de consumo 14,15,16,17,18.

No Brasil, os estudos que utilizaram o QQFA como método de avaliação da ingestão alimentar habitual de gestantes empregaram questionários originalmente construídos e validados para populações distintas. Nas investigações conduzidos por Barros et al. 19 e por Fonseca et al. 20, utilizouse o QQFA construído e validado para adultos não gestantes 21 , fato que limita a acurácia das informações obtidas.

O objetivo do presente estudo foi elaborar um questionário quantitativo de freqüência alimentar específico para gestantes, usuárias do Sistema Único de Saúde do Município de Ribeirão Preto, São Paulo, Brasil.

\section{Metodologia}

\section{Delineamento e população de estudo}

Estudo com desenho transversal, conduzido em Ribeirão Preto, interior de São Paulo. O município localiza-se a $313 \mathrm{~km}$ da capital, com população estimada em 563.105 habitantes, segundo estimativa populacional divulgada pelo IBGE em 2009 (Instituto Brasileiro de Geografia e Estatística. http://www.ibge.gov.br/, acessado em 23/ Nov/2009). A economia baseia-se no agronegó- cio, comércio e prestação de serviços, além da pesquisa universitária. Como indicadores, temse Índice de Desenvolvimento Humano (IDH) de 0,855 e taxa de mortalidade infantil de 10,47 por mil nascidos vivos.

O estudo foi conduzido com 150 gestantes usuárias do SUS do município, tamanho amostral estimado para investigação de diferenças de consumo de nutrientes de interesse no segundo trimestre gestacional. O tamanho amostral empregado em estudos de desenvolvimento de QQFA varia amplamente, de 100 a 1.477 indivíduos, a maioria deles contando com amostras na faixa de 100 a 200 pessoas 22,23,24,25,26,27. Foram utilizados como critérios de inclusão: idade entre 18 e 35 anos, índice de massa corporal prégestacional entre 19,8 e $26 \mathrm{~kg} / \mathrm{m}^{2} 28$, ausência de diabetes gestacional, cardiopatias, nefropatias, hipertensão arterial ou relato de outras patologias que pudessem alterar o consumo alimentar habitual.

\section{Coleta de dados}

A coleta de dados foi realizada de junho a outubro/2008, em unidades básicas de saúde das cinco regiões do município (Norte, Sul, Leste, Oeste e Central), por uma nutricionista e uma aluna de graduação em atividade de iniciação científica, previamente treinadas. $\mathrm{O}$ atendimento de gestantes é realizado diariamente nas unidades, e a coleta de dados foi feita estabelecendo-se esquema de plantão nas unidades selecionadas, em dias arbitrários. Nos dias de coleta de dados, todas as gestantes agendadas para o acompanhamento pré-natal foram convidadas a participar do estudo.

\section{Características sócio-demográficas}

Dados de idade, escolaridade e posse de bens de consumo foram obtidos por meio de questionário estruturado. Para a classificação econômica, foi escolhido o Critério de Classificação Econômica Brasil (CCEB), que permite estratificar a população em oito classes econômicas, baseandose nas respostas do entrevistado quanto à posse de bens, presença de empregada mensalista e grau de instrução do chefe da família. A classificação em pontos permite inferir-se sobre a renda familiar média 29.

\section{Avaliação antropométrica e idade gestacional}

O cálculo da idade gestacional foi realizado com base nos dados da ultrassonografia, quando presentes, ou na descrição da gestante, após relato quanto à data da última menstruação. A gesta- 
ção foi descrita em semanas gestacionais. Foram categorizadas no primeiro trimestre as gestantes entre 4 e 12 semanas; no segundo, aquelas entre 12 e 24 semanas; no terceiro, as mulheres entre 24 e 38 semanas de gestação. O peso prégestacional foi obtido consultando-se o registro no cartão da gestante; o peso no momento da entrevista foi aferido por meio de balança eletrônica digital modelo MEA 07700 (Plenna, São Paulo, Brasil), com capacidade de $150 \mathrm{~kg}$ e graduação de $100 \mathrm{~g}$. A altura foi medida utilizando-se uma fita métrica inextensível fixada na parede. O índice de massa corporal (IMC) pré-gestacional foi obtido dividindo-se o peso pré-gestacional pela estatura ao quadrado. Foram empregados os critérios do Institute of Medicine 28 para a avaliação da adequação de IMC pré-gestacional.

\section{Avaliação da ingestão alimentar}

Um inquérito recordatório de 24 horas (IR24h) foi obtido de 150 gestantes, no qual foram relatados todos os alimentos e bebidas consumidos no dia anterior à entrevista, bem como a quantidade ingerida em medidas caseiras, a forma de preparação do alimento, horário de consumo, detalhes referentes à marca e às características do produto, além de petiscos consumidos fora das refeições. As entrevistadoras foram previamente treinadas quanto às técnicas gerais de coleta de dados, conduta pessoal e forma de condução da entrevista. Este primeiro inquérito foi coletado pessoalmente no dia da coleta de dados.

Para fins de correção da distribuição da estimativa de nutrientes quanto à variabilidade intrapessoal, é recomendado que se utilizem duas medidas independentes da dieta, de uma amostra de pelo menos $20 \%$ da população de estudo 30 .

Dessa forma, obteve-se um segundo IR24h de uma subamostra de 90 gestantes, sendo 30 em cada trimestre gestacional. Essa subamostra foi determinada por sorteio simples estratificado por trimestre de gestação, e os inquéritos foram coletados entre 7 e 14 dias após a coleta do primeiro IR24h, por meio de contato telefônico 31 .

As quantidades dos alimentos, citadas nos IR24h em medidas caseiras, foram convertidas em gramas ou mililitros para a definição do tamanho das porções alimentares de referência 32

\section{Elaboração da lista de alimentos}

No total, 240 IR24h foram obtidos, tendo sido citados 305 alimentos. Estes foram agrupados em 104 itens, de acordo com a similaridade do valor nutricional para os nutrientes de interesse durante o período gestacional 7,8: energia, carboidratos, proteínas, lipídeos totais, ácidos graxos saturados, monoinsaturados, poliinsaturados, linoléico, linolênico, oléico, araquidônico, docosahexaenóico e eicosapentanóico, colesterol, fibras, cálcio, ferro, folato, cobre, magnésio, zinco, além das vitaminas: tiamina, riboflavina, vitamina A (UI), B12, B6, C e vitamina E. Modelos de regressão linear múltipla stepwise foram empregados para a redução da lista de alimentos, e a estimativa de cada nutriente de interesse foi a variável dependente após ajuste pela variância intra-individual e energia. Os alimentos de maior contribuição na variância interindividual para energia foram inseridos no modelo em ordem decrescente até que se atingisse $90 \%$. Os alimentos de maior contribuição na variância interindividual para os outros nutrientes foram então inseridos até que se atingisse o ponto de corte estipulado de $70 \%$ para todos os nutrientes selecionados 13. Assim, identificaram-se os alimentos que explicaram a maior variância interindividual da estimativa de cada nutriente de interesse. Esse procedimento originou uma primeira lista de alimentos, à qual foram posteriormente adicionados os alimentos fontes de nutrientes de interesse não citados nos IR24h, resultando em uma lista final com 85 itens alimentares.

\section{Determinação das porções alimentares}

As porções alimentares foram determinadas para gestantes em cada trimestre gestacional separadamente e para todas as gestantes, de acordo com a distribuição percentual dos pesos equivalentes às medidas caseiras referidas nos IR24h. A porção mediana foi utilizada como referência (percentil 50) para cada item alimentar, atribuindo-se os conceitos pequena, grande e extragrande quando os percentis foram 25, 75 e 100, respectivamente. Após verificação de diferenças de porções consumidas segundo trimestre gestacional, foi elaborada uma proposta de porções para cada item baseada nos percentis de consumo do grupo como um todo, incorporando-se a variação de consumo em cada trimestre gestacional. Para os alimentos que não foram citados nos inquéritos alimentares e posteriormente foram incluídos no QQFA por serem fontes de nutrientes de interesse, utilizou-se como referência a porção mediana descrita na literatura 32 .

A versão final do QQFA inclui: os alimentos, as opções de freqüência de consumo (diária, semanal, mensal ou durante o período gestacional), o número de vezes que a participante consome aquele alimento, a porção mediana - em medidas caseiras e em $\mathrm{g} / \mathrm{mL}$ - e o tamanho da porção da participante. Foram incluídas questões sobre a forma de preparo dos alimentos (fritos 
ou assados, retirada da gordura visível das carnes), especificidades do teor de gordura para os laticínios (integral ou desnatado), teor de fibras de cereais (grãos integrais ou refinados), tipos de molho consumido com massas, tipo de tempero adicionado às saladas e adição de açúcar simples às bebidas preparadas.

Um estudo piloto foi conduzido com 50 gestantes adultas usuárias de unidades básicas de saúde (UBS) do município, em todos os trimestres gestacionais, com o objetivo de avaliar a consistência do tamanho das porções, a forma de apresentação do questionário e a compreensão das participantes.

\section{Análise dos dados}

A normalidade das variáveis dietéticas foi avaliada por meio do teste de Kolmogorov-Smirnov. As variáveis que não apresentaram distribuição normal foram transformadas por meio do logaritmo natural, sendo sua normalidade novamente testada.

Para o ajuste das estimativas dos nutrientes quanto à variabilidade intra-individual, a análise de variância pelo teste de ANOVA em cada trimestre gestacional foi empregada em subamostra de gestantes com dados de dois IR24h. Os valores de variabilidade obtidos pela análise da subamostra foram empregados para todas as mulheres do mesmo trimestre de gestação. Os nutrientes foram ajustados considerando-se a média de ingestão do grupo (cada trimestre), o consumo individual e a razão entre o desvio-padrão interpessoal e o desvio-padrão observado 33 . $\mathrm{O}$ ajuste dos nutrientes pelas calorias totais foi feito pelo método residual, considerando-se a estimativa de energia como variável dependente e os nutrientes como variáveis independentes, em modelos de regressão linear simples. Os valores dos resíduos não padronizados gerados pelos modelos de regressão foram somados ao consumo esperado do nutriente para a média da ingestão calórica da população estudada, obtendo-se os valores de nutrientes ajustados pelas calorias totais 34 . Posteriormente aos ajustes pela variabilidade intra-individual e energia, as variáveis dietéticas foram reconvertidas de seu logaritmo natural para a unidade de origem pelo cálculo de seu exponencial.

A análise do valor nutricional obtido dos inquéritos foi processada com o auxílio do programa NutWin versão 1.5 (Escola Paulista de Medicina, Universidade Federal de São Paulo, São Paulo, Brasil), empregando-se as tabelas da USDA (United States Department of Agriculture) 35, suplementada pela Tabela Brasileira de Composição de Alimentos (TACO) 36 .
A comparação entre trimestres para variáveis contínuas com distribuição normal foi realizada por meio do teste de ANOVA; para variáveis sem distribuição normal, pelo teste de Kruskal-Wallis; e para variáveis categóricas, por meio do teste de $\chi^{2}$. Os valores foram expressos em média e desvio-padrão (DP), mediana ou freqüência. Significância estatística foi estabelecida no nível de $\mathrm{p}<0,05$. As análises estatísticas foram feitas com o SPSS 17.0 (SPSS Inc., Chicago, Estados Unidos).

\section{Aspectos éticos}

O projeto foi aprovado pelo Comitê de Ética em Pesquisa do Centro de Saúde Escola da Faculdade de Medicina de Ribeirão Preto, da Universidade de São Paulo, e sua execução foi autorizada pela Secretaria Municipal de Saúde. Às participantes, foi entregue carta de esclarecimento, e a participação no estudo se deu mediante assinatura do Termo de Consentimento Livre e Esclarecido

O presente estudo foi desenvolvido de acordo com os princípios éticos contidos na Declaração de Helsinki (1964, reformulada em 1975, 1983, 1989, 1996 e 2000), da World Medical Association, e atende à legislação do Comitê de Ética em Pesquisa da Universidade Federal de São Paulo e do Comitê de Ética em Pesquisa do Centro de Saúde Escola da Faculdade de Medicina de Ribeirão Preto.

\section{Resultados}

No total, 224 gestantes foram convidadas a participar do estudo e 217 aceitaram. Destas, 67 foram excluídas: 54 (80,7\%) com idade superior ou inferior aos critérios estabelecidos, 10 (14,9\%) devido aos critérios de adequação de IMC prégestacional e $3(4,4 \%)$ pela presença de comorbidades. Desta forma, a amostra foi composta por 150 gestantes.

A Tabela 1 apresenta as características sociodemográficas, estado nutricional e estilo de vida das gestantes, conforme trimestre gestacional. Não foram observadas diferenças quanto à idade, peso pré-gestacional, IMC pré-gestacional, escolaridade ou condição sócio-econômica, conforme trimestre gestacional. Como esperado, houve diferença para ganho de peso gestacional ( $\mathrm{p}<0,001)$ e IMC atual ( $\mathrm{p}<0,001)$.

A lista final com 85 alimentos representa a variabilidade interindividual de $98,4 \%$ da energia; $88,3 \%$ de carboidratos; $77,2 \%$ de proteínas; $87,4 \%$ de lipídeos totais; $87,5 \%$ de ácidos graxos saturados; $86,4 \%$ ácidos graxos monoinsaturados; $78,1 \%$ de ácidos graxos poliinsaturados; $76,5 \%$ de ácido graxo linoléico; $74,2 \%$ de ácido graxo lino- 
Tabela 1

Características sócio-demográficas, estado nutricional e estilo de vida das gestantes, conforme trimestre gestacional. Ribeirão Preto, São Paulo, Brasil, 2008. ( $=150)$.

\begin{tabular}{|c|c|c|c|c|}
\hline Variáveis & $\begin{array}{c}\text { 1ㅇ trimestre } \\
(n=50)\end{array}$ & $\begin{array}{c}\text { 2o trimestre } \\
\qquad(n=50)\end{array}$ & $\begin{array}{c}\text { 3o trimestre } \\
(n=50)\end{array}$ & Valor de $p$ * \\
\hline & \multicolumn{4}{|c|}{ Média \pm desvio-padrão } \\
\hline Idade (anos) & $24 \pm 4$ & $24 \pm 4$ & $24 \pm 5$ & 0,91 \\
\hline Peso pré-gestacional (kg) & $56,9 \pm 7,7$ & $55,9 \pm 6,9$ & $56,2 \pm 7,3$ & 0,77 \\
\hline IMC pré-gestacional (kg/m²) & $21,9 \pm 2,4$ & $21,4 \pm 2,3$ & $21,8 \pm 2,5$ & 0,58 \\
\hline IMC atual $\left(\mathrm{kg} / \mathrm{m}^{2}\right)$ & $22,8 \pm 3,7$ & $23,4 \pm 4,5$ & $26,6 \pm 2,8$ & $<0,001$ \\
\hline \multirow[t]{2}{*}{ Ganho de peso gestacional (kg) } & $3,2 \pm 3,9$ & $6,2 \pm 5,2$ & $11,7 \pm 4,4$ & $<0,001$ \\
\hline & \multicolumn{4}{|c|}{ Número (freqüência) } \\
\hline \multicolumn{5}{|l|}{ Escolaridade (anos de estudo) } \\
\hline Até 4 & $3(6)$ & $3(6)$ & $4(8)$ & \\
\hline $4-8$ & $9(18)$ & $9(18)$ & $11(22)$ & 0,743 \\
\hline Mais de 8 & $38(76)$ & $38(76)$ & $35(70)$ & \\
\hline \multicolumn{5}{|l|}{ Classe sócio-econômica } \\
\hline A & - & $1(2)$ & - & \\
\hline B & $4(8)$ & $12(24)$ & $9(18)$ & \\
\hline $\mathrm{C}$ & $37(74)$ & $30(60)$ & $28(56)$ & 0,146 \\
\hline $\mathrm{D}$ & $8(16)$ & $6(12)$ & $13(26)$ & \\
\hline$E$ & $1(2)$ & $1(2)$ & - & \\
\hline Nunca consomem bebidas alcoólicas & $43(86)$ & $47(94)$ & $47(94)$ & 0,65 \\
\hline Tabagismo & $0(0)$ & $2(4)$ & $2(4)$ & 0,36 \\
\hline
\end{tabular}

* Segundo teste de ANOVA para variáveis contínuas e $\chi^{2}$ para variáveis categóricas.

lênico; $80,4 \%$ de colesterol; $79 \%$ de fibras; $95,2 \%$ de cálcio; $94,9 \%$ de ferro; $91,7 \%$ de folato; $95,5 \%$ de magnésio; $98,8 \%$ de vitamina A (UI); $85,2 \%$ de vitamina C; $89 \%$ de vitamina B12; $88,3 \%$ de cobre; $95,2 \%$ de zinco; $99,1 \%$ de tiamina; $94,3 \%$ de riboflavina; $94,7 \%$ de vitamina B6 e $94,7 \%$ de vitamina $\mathrm{E}$.

A Tabela 2 traz os itens alimentares que compuseram o QQFA, bem como as porções medianas, em gramas e medidas caseiras, conforme trimestre gestacional e para todas as gestantes. Alguns alimentos não citados nos IR24h foram posteriormente incluídos na lista de alimentos, por causa da sua contribuição em nutrientes de interesse: abacate, oleaginosas, frutos do mar e atum, por serem importantes fontes de ácidos graxos monoinsaturados e poliinsaturados; arroz e pães integrais, por serem importantes fontes de fibra da dieta. Houve diferença no tamanho das porções de doces à base de leite $(p=0,015)$ e tomate $(p=0,048)$, conforme trimestres gestacionais. Não se verificaram diferenças para os demais itens da lista de alimentos.

Após estudo-piloto conduzido entre 50 gestantes adultas usuárias de UBS do município, foram alteradas a ordem e a apresentação da lista de alimentos, bem como a forma de condução das perguntas.

\section{Discussão}

Para a investigação da relação entre ingestão alimentar habitual e desfechos de saúde, são necessários métodos adequados aos objetivos do estudo e às características da população-alvo. Há evidências crescentes da influência da ingestão alimentar habitual durante a gestação sobre desfechos maternos e infantis 7,8. O QQFA é considerado um método adequado para investigação da relação entre ingestão alimentar habitual e desfechos de saúde em estudos epidemiológicos 14 . Entretanto, desconhecemos a existência desse instrumento desenvolvido e validado especificamente para a população gestante no Brasil.

No presente estudo, a definição dos critérios de inclusão objetivou padronizar a população de estudo a fim de limitar a seleção de mulheres com fatores de risco conhecidos para morbidades na gestação ou com outros fatores que alterassem o consumo alimentar. Assim, foram selecionadas gestantes com idade entre 18 e 35 
Porção mediana (em gramas) dos itens alimentares do Questionário Quantitativo de Freqüência Alimentar (QQFA), conforme trimestre gestacional. Ribeirão Preto, São Paulo, Brasil, 2008. ( $N=150)$.

\begin{tabular}{|c|c|c|c|c|c|c|}
\hline & $\begin{array}{l}\text { 1o trimestre } \\
\text { gestacional }\end{array}$ & $\begin{array}{l}\text { 2o trimestre } \\
\text { gestacional }\end{array}$ & $\begin{array}{l}\text { 3o trimestre } \\
\text { gestacional }\end{array}$ & Valor de $p$ * & $\begin{array}{c}\text { Valor } \\
\text { mediano } \\
\text { para todas } \\
\text { as gestantes }\end{array}$ & Medida caseira \\
\hline \multicolumn{7}{|l|}{ Média \pm desvio-padrão } \\
\hline Abacate ou abacatada ** & - & - & - & - & 147,5 & 1 fatia média \\
\hline Abacaxi & 200 & - & - & - & 200 & 2 fatias médias \\
\hline Abóbora cozida & 200 & 84 & 135 & 0,543 & 135 & 1 pires \\
\hline Abobrinha cozida & 75 & 90 & 90 & 0,607 & 72 & 1 unidade pequena \\
\hline Acelga, alface ou repolho (cru ou cozido) & 45 & 50 & 40 & 0,685 & 36 & 1 prato de sobremesa \\
\hline Achocolatado ou cappuccino & 30 & 20 & 20 & 0,648 & 22 & 2 colheres de sobremesa \\
\hline Açúcar (adicionado em bebidas) & 15 & 15 & 10 & 0,412 & 16 & 1 colher de sobremesa \\
\hline Agrião, almeirão, rúcula ou couve & 40 & 40 & 50 & 0,581 & 50 & 1 prato cheio \\
\hline Arroz branco & 165 & 180 & 180 & 0,608 & 170 & 2 escumadeiras médias cheias \\
\hline Arroz integral ** & - & - & - & - & 134 & 1 colher de arroz cheia \\
\hline Atum ** & - & - & - & - & 32 & 2 colheres de sopa cheias \\
\hline Banana & 80 & 80 & 80 & - & 80 & 1 unidade média \\
\hline Bacon ou torresmo & 106 & 65 & 209 & 0,269 & 100 & 6 fatias médias \\
\hline Beterraba (crua/cozida) & 125 & 48 & 36 & 0,248 & 52 & 2 fatias grandes \\
\hline Bolacha doce com recheio & 90 & 90 & 82,5 & 0,978 & 87,5 & 7 unidades \\
\hline Bolacha doce sem recheio & 30 & 53 & 100 & 0,527 & 50 & 10 unidades \\
\hline Bolo & 120 & 105 & 120 & 0,504 & 100 & 1 fatia grande \\
\hline Brócolis & 30 & 35 & 30 & 0,438 & 30 & 1 ramo \\
\hline Café & 50 & 50 & 50 & - & 50 & 1 xícara de café \\
\hline Cenoura (crua/cozida) & 12 & - & 90 & 0,317 & 30 & 2 colheres de sopa cheias \\
\hline Carne bovina frita/Carne de panela & 110 & 110 & 110 & - & 100 & $\begin{array}{c}1 \text { filé médio ou } 3 \text { pedaços } \\
\text { médios }\end{array}$ \\
\hline Carne bovina assada (bife grelhado/Carne moída) & 180 & 120 & 125 & 0,518 & 100 & $\begin{array}{c}1 \text { filé médio ou } 4 \text { colheres de } \\
\text { sopa cheias }\end{array}$ \\
\hline \multicolumn{7}{|l|}{ Carne de porco } \\
\hline Pernil/Lombo & 90 & 132,5 & 175 & 0,266 & 150 & 1 filé médio \\
\hline Lingüiça & 60 & 75 & 120 & 0,107 & 60 & 1 gomo \\
\hline Bisteca & - & - & - & & 100 & 1 filé médio \\
\hline Castanhas, nozes, amendoim ** & - & - & - & - & 20 & 8 unidades \\
\hline Chocolate & 30 & 30 & 30 & - & 30 & 1 bombom \\
\hline Doces com frutas/Picolé de frutas & 65 & 100 & 35 & 0,544 & 80 & $\begin{array}{l}2 \text { colheres de sopa cheias } \\
\text { ou } 1 \text { picolé }\end{array}$ \\
\hline Doce com leite & 91,5 & 99 & 43 & 0,015 & 69 & 1 fatia média \\
\hline Dobradinha & - & - & 70 & - & 97,5 & 3 colheres de sopa cheias \\
\hline Feijão cozido & 156 & 156 & 156 & - & 156 & 2 conchas médias \\
\hline $\begin{array}{l}\text { Feijão temperado (feijoada, com lingüiça } \\
\text { ou bacon) }\end{array}$ & 240 & 480 & 337 & 0,407 & 273 & $31 / 2$ conchas \\
\hline Fígado/Moela & 30 & 30 & - & - & 30 & 1 unidade média \\
\hline Frango assado & 75 & 128 & 100 & 0,139 & 180 & 1 filé médio \\
\hline Frango frito & 172 & 71 & 120 & 0,193 & 180 & 1 filé médio \\
\hline $\begin{array}{l}\text { Frango xadrez, estrogonofe de frango, } \\
\text { fricassê de frango }\end{array}$ & 120 & - & - & - & 120 & 2 colheres de sopa cheias \\
\hline Frutos do mar $* \star$ & - & - & - & - & 100 & 5 colheres de sopa cheias \\
\hline Geléia, mel ou melado & 28 & - & - & - & 10 & 1 colher de sobremesa \\
\hline Goiaba & 340 & - & - & - & 340 & 2 unidades médias \\
\hline
\end{tabular}

(continua) 


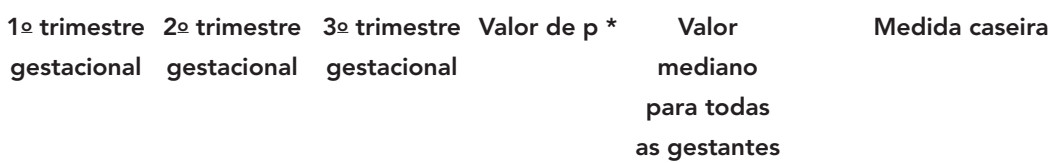

\begin{tabular}{|c|c|c|c|c|c|c|}
\hline logurte desnatado & 120 & 120 & 93 & 0,475 & 150 & 1 copo americano \\
\hline $\begin{array}{l}\text { logurte integral (coalhada, iogurte natural } \\
\text { ou de frutas) }\end{array}$ & 200 & 200 & 200 & - & 250 & 1 pote \\
\hline Lanches (cachorro quente, hambúrguer) & 125 & 116,5 & 125 & 0,849 & 125 & 1 unidade \\
\hline Laranja/Tangerina & 170 & 320 & 160 & 0,205 & 160 & 1 unidade média \\
\hline Lasanha/Massas recheadas com carne & 120 & 210 & 165 & 0,189 & 122,5 & $\begin{array}{l}1 \text { escumadeira rasa ou } 1 \\
\text { pedaço pequeno }\end{array}$ \\
\hline Leite integral/Desnatado & 250 & 250 & 250 & - & 250 & 1 copo de requeijão cheio \\
\hline Legumes refogados & 91 & 156 & - & 0,564 & 90 & 1 escumadeira média cheia \\
\hline Macarrão/Outras massas & 225 & 277 & 135 & 0,867 & 220 & 2 escumadeiras médias cheias \\
\hline Macarrão tipo miojo & 80 & 80 & 80 & - & 80 & 1 pacote \\
\hline Maçã/Pêra & 93 & 93 & 93 & - & 93 & 1 unidade média \\
\hline $\begin{array}{l}\text { Mandioca, batata ou purê de batata ou } \\
\text { mandioquinha (frita/cozida) }\end{array}$ & 37 & 100 & 173 & 0,128 & 95 & 1 escumadeira média rasa \\
\hline Manga/Caqui & 180 & - & 90 & - & 180 & 1 unidade média \\
\hline Mamão & 221 & - & 136 & 0,150 & 170 & 1 fatia média \\
\hline Manteiga & 5 & 5 & 5 & - & 5 & 1 ponta de faca \\
\hline Margarina & 5 & 10 & 5 & 0,066 & 5 & 1 ponta de faca \\
\hline Melancia/Melão & 200 & 150 & 200 & 0.154 & 200 & 1 fatia média \\
\hline Milho verde & 80 & 150 & - & - & 100 & $\begin{array}{c}4 \text { colheres de sopa cheias } \\
\text { ou } 1 \text { espiga }\end{array}$ \\
\hline Mingau & 200 & 200 & - & 0,151 & 200 & 1 prato fundo raso \\
\hline Morango & 48 & 60 & 84 & 1,000 & 108 & 9 unidades \\
\hline $\begin{array}{l}\text { Mortadela, salame, presunto, peito } \\
\text { de peru ou salsicha }\end{array}$ & 35 & 124 & 100 & 0,312 & 40 & 2 fatias médias \\
\hline Ovo/Omelete & 48 & 48 & - & - & 50 & 1 unidade \\
\hline Paçoca/Pé-de-moleque & 90 & 30 & 52 & 0,259 & 60 & 2 unidades \\
\hline Pão francês/Pão de fôrma & 52 & 100 & 100 & 0,618 & 50 & 1 unidade \\
\hline Pão integral ** & - & - & - & - & 50 & 2 fatias \\
\hline Peixe cozido & 300 & 60 & 200 & 0,252 & 100 & 1 filé médio \\
\hline Peixe frito & 150 & 65 & 137 & 0,119 & 100 & 1 filé médio \\
\hline Pepino & 94,5 & 126 & - & 0,439 & 120 & 2 pires cheios \\
\hline Pêssego & 100 & 630 & - & 0,317 & 300 & 3 unidades médias \\
\hline Pizza & 240 & 420 & 241 & 0,723 & 210 & 2 fatias médias \\
\hline $\begin{array}{l}\text { Queijos amarelos (parmesão, mussarela, } \\
\text { provolone, queijo prato) }\end{array}$ & 45 & 30 & 25 & 0,098 & 30 & 3 fatias médias \\
\hline Queijo branco (fresco, ricota) & 175 & 50 & 50 & 0,803 & 50 & 2 fatias médias \\
\hline Requeijão & 18 & 9 & 6 & 0,624 & $10 / 45$ & $\begin{array}{l}1 \text { ponta de faca/ } \\
1 \text { fatia média }\end{array}$ \\
\hline Risoto, arroz carreteiro ou arroz à grega, canja & - & 115 & 135 & - & 134 & 1 colher de arroz cheia \\
\hline Rosca doce/Sonho & 50 & 50 & 90 & 0,567 & 100 & 1 fatia grande \\
\hline Salgado assado & 110 & 110 & 110 & - & 80 & 1 unidade média \\
\hline Salgado frito & 100 & 180 & 50 & 0,027 & 100 & 1 unidade grande \\
\hline Salgado tipo chips ou pipoca & 96 & 100 & 100 & 0,584 & 96 & 1 pacote \\
\hline Sardinha & - & - & - & - & 82 & 2 unidades \\
\hline Sopa de legumes & 467 & 310 & 362 & 0,177 & 310 & 1 prato fundo \\
\hline Sorvete de massa & 91 & 108 & - & - & 100 & 1 bola grande \\
\hline Suco de laranja natural & 250 & 250 & 250 & - & 250 & 1 copo de requeijão cheio \\
\hline Suco de outras frutas (natural) & 250 & 250 & 250 & - & 250 & 1 copo de requeijão cheio \\
\hline
\end{tabular}

(continua) 


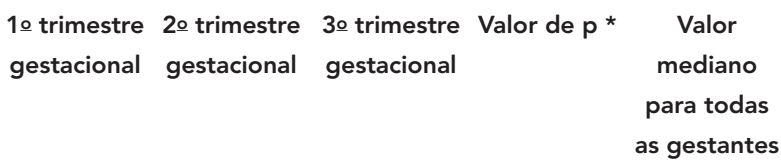

Valor

mediano

para todas

as gestantes

\begin{tabular}{|c|c|c|c|c|c|c|}
\hline Suco artificial/Refrigerante & 275 & 250 & 250 & 0,776 & 250 & 1 copo de requeijão cheio \\
\hline $\begin{array}{l}\text { Estrogonofe de carne, bife à role, } \\
\text { carne com legumes }\end{array}$ & 106 & 92 & 85 & 0,861 & 80 & 2 colheres de arroz cheias \\
\hline Tomate & 72 & 90 & 90 & 0,048 & 90 & 1 unidade média \\
\hline Torrada/Bolacha salgada/Biscoito de polvilho & 33 & 35 & 49 & 0,323 & 33 & 5 unidades \\
\hline Uva & 170 & - & 170 & 0,653 & 170 & 1 cacho pequeno \\
\hline Vitamina de fruta com leite & 375 & 500 & 250 & 0,843 & 300 & 1 copo grande \\
\hline
\end{tabular}

* Teste de Kruskal-Wallis para investigação de diferenças conforme trimestres gestacionais;

** Alimentos inseridos posteriormente ao questionário.

anos, considerando-se as distintas recomendações nutricionais na adolescência 37 e também o risco de hipertensão, diabetes, placenta prévia e complicações da gestação em mulheres acima dos 35 anos de idade 38 . O IMC pré-gestacional acima dos valores normais é fator de risco para complicações, como pré-eclampsia e diabetes gestacional 4,38,39,40. Além disso, indivíduos portadores de excesso de peso tendem a sub-relatar o consumo de alimentos, o que poderia comprometer a confiabilidade dos dados 41,42,43.

As gestantes avaliadas apresentaram idade média de 24 anos e eutrofia no período pré-gestacional. Verificou-se predominância de mulheres com pelo menos oito anos de estudo, pertencentes à classe sócio-econômica $\mathrm{C}$, segundo o critério de classificação econômica do Brasil 29.

A metodologia empregada para o desenvolvimento do questionário é semelhante à descrita para versões construídas para outras populações e diferentes objetivos 22,23,24,25,26,27. Contudo, no presente estudo, as estimativas de nutrientes de interesse dos IR24h foram ajustadas pela variabilidade intra-individual e energia, para a determinação da lista de alimentos. O emprego de um único IR24h não representa o que um indivíduo ingere habitualmente em face da elevada variabilidade intra-individual do consumo de alimentos e, portanto, da estimativa de nutrientes 44 .

$\mathrm{O}$ ajuste pela variabilidade intra-individual dos nutrientes resulta em uma distribuição de ingestão habitual que melhor refletirá a variabilidade interindividual. Para este ajuste, pode-se empregar dados de variabilidade intra-individual de populações externas, utilizar dados de dois IR24h de toda a população, ou obter um segundo IR24h de uma subamostra (pelo menos $20 \%$ da amostra total), a fim de se determinarem os valores de variabilidade que serão empregados para correção dos dados de toda a amostra estudada 30. Em adição, nesta investigação, as estimativas de nutrientes foram ajustadas pela energia. Essa estratégia permite a obtenção de uma lista de alimentos que reflita a maior variabilidade interindividual de nutrientes, independente da energia da dieta das mulheres 13 , porém a competência do QQFA desenvolvido somente poderá ser testada por meio das análises de reprodutibilidade e validação.

Verificou-se pouca variedade na dieta das mulheres avaliadas, o que pode ser constatado pelo número reduzido de itens alimentares relatados nos IR24h, independente do período de gestação da mulher. Esse fato pode ser explicado pela homogeneidade da amostra em relação à condição sócio-econômica, faixa etária e sexo. Por sua vez, o número de itens alimentares citados no presente estudo foi semelhante ao verificado por Cardoso \& Stocco 23 (230 itens) e Furlan-Viebig \& Pastor-Valero 24 (314 itens), e inferior ao número de itens reportados em IR24h por Fisberg et al. 27 (1.040 itens), em estudo de base populacional para desenvolvimento de QQFA 27. Este fato pode ser atribuído à grande heterogeneidade de faixa etária (entre 20 e 101 anos), renda e sexo, além do elevado tamanho amostral desta última investigação $(n=1.477)$.

Um artigo de revisão desenvolvido por Cade et al. 14, analisando artigos publicados sobre validação de QQFA, constatou que o número de itens alimentares das listas empregadas variou de cinco a 350 itens, com mediana de 79 itens 14 . A utilização de QQFA com listas extensas pode comprometer a memória e a adesão dos participantes, afetando, dessa maneira, a confiabilidade das informações obtidas. A lista de alimentos desenvolvida no presente estudo é semelhante à de outros QQFA desenvolvidos e validados para 
adultos no Brasil 21,23, entretanto o tamanho das porções é sistematicamente maior.

A definição da lista de alimentos e do tamanho das porções de um QQFA com base nos hábitos de consumo da população a ser investigada é capaz de minimizar a ocorrência de erros sistemáticos de medida 13,14. A lista de alimentos do QQFA elaborada para esta pesquisa respondeu por grande parte da variabilidade interindividual da estimativa de nutrientes de interesse para o período gestacional. O fato de não termos encontrado diferenças importantes no tamanho das porções para a maioria dos alimentos conforme os trimestres gestacionais sugere que a lista de alimentos do QQFA construído pode ser capaz de avaliar o consumo alimentar no período gestacional como um todo.

As principais limitações do presente estudo estão relacionadas à metodologia empregada para a obtenção dos IR24h e à carência do uso de ilustrações para padronização do tamanho da porção do alimento consumido. A metodologia de passagens múltiplas tem sido recomendada como procedimento de escolha para obtenção de IR24h 45 , mas esta não foi empregada no presente estudo. Os IR24h foram obtidos em dias arbitrários, sem controle para representação quanto aos dias da semana. Além disso, apesar de o estudo ter sido desenvolvido nas cinco regiões do município, a amostra selecionada não pode ser considerada como representativa deste. Cabe destacar a dificuldade na obtenção de amostras aleatórias de gestantes, devido à falta de dados atualizados quanto ao número de nascidos vivos e gestantes acompanhadas em cada unidade básica de saúde da cidade.

Sabe-se, ainda, que o delineamento longitudinal do estudo seria mais indicado para avaliar diferenças no consumo alimentar habitual segundo período gestacional. O IMC foi avaliado de acordo com os critérios do Institute of Medicine de 1990 28, disponível na ocasião da coleta de dados. Dentre as vantagens, destacam-se a inclusão de mulheres em todos os períodos da gestação e a investigação de diferenças de tamanho de porção de alimentos conforme os trimestres. Ademais, a lista de alimentos foi desenvolvida enfocando-se diversos nutrientes de interesse para investigação da relação entre ingestão alimentar habitual materna e desfechos maternos e infantis.

Este estudo é pioneiro no Brasil. As análises quanto à reprodutibilidade e validação relativa do QQFA estão em andamento e permitirão a avaliação da acurácia do questionário para investigação da ingestão alimentar habitual desse grupo populacional.

\section{Resumo}

O objetivo do presente estudo foi desenvolver um questionário quantitativo de freqüencia alimentar para gestantes adultas usuárias do Sistema Único de Saúde de Ribeirão Preto, São Paulo, Brasil. Um inquérito recordatório de 24 horas (IR24h) foi obtido de 150 gestantes, 50 em cada trimestre gestacional, com idade entre 18 e 35 anos. Um segundo IR24h foi obtido de 90 gestantes, 30 em cada trimestre gestacional, entre 7 e 15 dias após o primeiro inquérito. Uma lista de 85 alimentos foi estabelecida por regressão linear múltipla stepwise, estimando-se a contribuição percentual para a variabilidade interindividual da estimativa de energia e 28 nutrientes de interesse, com contribuição de 74,2 a 98,8\% para os nutrientes de interesse selecionados. As porções alimentares foram determinadas de acordo com a distribuição percentual dos pesos relatados e descritas nos percentis 25, 50, 75 e 100. Desconhecemos a existência de questionário quantitativo de freqüência alimentar desenvolvido para gestantes no Brasil.

Ingestão de Alimentos; Gestantes; Epidemiologia Nutricional 


\section{Colaboradores}

T. Oliveira foi responsável pela coleta, análise e interpretação dos resultados; elaborou a primeira versão do manuscrito. F. D. Marquitti foi responsável pela coleta, análise e interpretação dos resultados; revisou criticamente o manuscrito. M. A. B. L. Carvalhaes foi responsável pela interpretação dos resultados e revisou criticamente o manuscrito. D. S. Sartorelli foi responsável pela interpretação dos resultados e elaboração do manuscrito

\section{Agradecimentos}

Este trabalho foi desenvolvido com o apoio financeiro da Fundação de Amparo à Pesquisa do Estado de São Paulo (FAPESP, processo no. 2008/51357-8) e do Programa Institucional de Bolsas de Iniciação Científica (PIBIC Santander, processo no ${ }^{\circ}$. 081471177).

\section{Referências}

1. Baião MR, Deslandes SF. Alimentação na gestação e puerpério. Rev Nutr 2006; 19:245-53.

2. Kramer MS. Determinants of low birth weight: methodological assessment and meta-analysis. Bull World Health Organ 1987; 65:663-737.

3. Kac G, Velásquez-Meléndez G. Ganho de peso gestacional e macrossomia em uma coorte de mães e filhos. J Pediatr (Rio J.) 2005; 81:47-53.

4. Nucci LB, Schimidt MI, Duncan BB, Fuchs SC, Fleck ET, Britto MMS. Nutritional status of pregnant women: prevalence and associated pregnancy outcomes. Rev Saúde Pública 2001; 35:502-7.

5. Wolfe $\mathrm{H}$. High prepregnancy body-mass index - A maternal-fetal risk factor. N England J Med 1998; 338:191-2.

6. Seligman LC, Duncan BB, Branchtein L, Galo DSM, Mengue SS, Schimidt MI. Obesity and gestational weight gain: cesarean delivery and labor complications. Rev Saúde Pública 2006; 40:457-65.

7. Costello AM, Osrin D. Micronutrient status during pregnancy and outcomes for newborn infants in developing countries. J Nutr 2003; 133(5 Suppl 2):1757-64.

8. Christian P. Micronutrients and reproductive health issues: an international perspective. J Nutr 2003; 133:1969-73.

9. Oken E, Ning YI, Shiman SLR, Edwards JWR, Olsen SF, Gillman MW. Diet during pregnancy and risk of preeclampsia or gestational hypertension. Ann Epidemiol 2007; 17:663-8.
10. Scholl TO, Hediger ML, Schall JI, Khoo CS, Fisher RL. Dietary and serum folate: their influence on the outcome of pregnancy. Am J Clin Nutr 1996; 63:520-5.

11. Clapp JF. Maternal carbohydrate intake and pregnancy outcome. Proc Nutr Soc 2002; 61:45-50.

12. Scholl TO, Chen X, Khoo CS, Lenders C. The dietary glycemic index during pregnancy: influence on infant birth weight, fetal growth, and biomarkers of carbohydrate metabolism. Am J Epidemiol 2004; 159:467-74.

13. Willet W. Food frequency methods. In: Willet W, editor. Nutritional epidemiology. 2nd Ed. Oxford: Oxford University Press; 1998. p. 74-100.

14. Cade J, Thompson R, Burley V, Warm D. Development, validation and utilization of food-frequency questionnaires - a review. Public Health Nutr 2002; 5:567-87.

15. Chen L, Hu FB, Yeung E, Willett W, Zhang C. Prospective study of pre-gravid sugar-sweetened beverage consumption and the risk of gestational diabetes mellitus. Diabetes Care 2009; 32:2236-41.

16. Zhang C, Liu S, Solomon CG, Hu FB. Dietary fiber intake, dietary glycemic load, and the risk for gestational diabetes mellitus. Diabetes Care 2006; 29:2223-30.

17. Radesky JS, Oken E, Rifas-Shiman SL, Kleinman KP, Rich-Edwards JW, Gillman MW. Diet during early pregnancy and development of gestational diabetes. Paediatr Perinat Epidemiol 2008; 22:47-59. 
18. Zhang C, Williams MA, Frederick IO, Jing IB, Sorensen TK, Kestin MM, et al. Vitamin C and the risk of gestational diabetes mellitus: a case-control study. J Reprod Med 2004; 49:257-66.

19. Barros DC, Pereira RS, Gama SGN, Leal MC. O consumo alimentar de gestantes adolescentes no $\mathrm{Mu}-$ nicípio do Rio de Janeiro. Cad Saúde Pública 2004; 20 Suppl 1:S121-9.

20. Fonseca VM, Sichieri R, Basílio R, Ribeiro LVC. Consumo de folato em gestantes de um hospital público do Rio de Janeiro. Rev Bras Epidemiol 2003; 6:319-27.

21. Sichieri R, Everhart JE. Validity of a brazilian food frequency questionnaire against dietary recalls and estimated energy intake. Nutr Res 1998; 18:1649-59.

22. Lima FEL, Fisberg RM, Slater B. Desenvolvimento de um questionário quantitativo de freqüência alimentar (QQFA) para um estudo caso-controle de dieta e câncer de mama em João Pessoa - PB. Rev Bras Epidemiol 2003; 6:373-9.

23. Cardoso MA, Stocco PR. Desenvolvimento de um questionário quantitativo de freqüência alimentar em imigrantes japoneses e seus descendentes residentes em São Paulo, Brasil. Cad Saúde Pública 2000; 16:107-14.

24. Furlan-Viebig R, Pastor-Valero M. Desenvolvimento de um questionário de freqüência alimentar para o estudo de dieta e doenças não transmissíveis. Rev Saúde Pública 2004; 38:581-4.

25. Collucci ACA, Philippi ST, Slater B. Desenvolvimento de um questionário de freqüência alimentar para avaliação do consumo alimentar de crianças de 2 a 5 anos de idade. Rev Bras Epidemiol 2004; 7:393-401.

26. Ribeiro AB, Cardoso MA. Construção de um questionário de freqüência alimentar como subsídio para programas de prevenção de doenças crônicas não transmissíveis. Rev Nutr PUCCAMP 2004; 15:239-45.

27. Fisberg RM, Colucci ACA, Morimoto JM, Marchioni DML. Questionário de freqüência alimentar para adultos com base em estudo populacional. Rev Saúde Pública 2008; 42:550-4.

28. Institute of Medicine. Nutrition during pregnancy. Part I: weight gain. Part II: nutrient supplements. Washington DC: National Academies Press, 1990.

29. Associação Brasileira de Empresas de Pesquisa. Dados com base no Levantamento sócio-econômico 2006 e 2007. http://www.abep.org (acessado em $28 / \operatorname{Jan} / 2008$ ).

30. National Academy of Sciences. Dietary reference intake: applications in dietary assessment. Washington DC: National Academic Press; 2000.

31. Tran KM, Johnson RK, Soultanakis R, Matthews DE. In-person vs telephone-administered multiple pass 24-hours recalls in women: validation with doubly labeled water. J Am Diet Assoc 2000; 100:777-83.

32. Pinheiro ABV, Lacerda EMA, Benzecry EH, Gomes MCS, Costa VM. Tabela para avaliação do consumo alimentar em medidas caseiras. 5ạ Ed. Rio de Janeiro: Editora Atheneu; 2004.
33. Fisberg RM, Slater B, Marchioni DML, Martini LA. Anexo 7 - Ajuste de dados de energia e nutrientes para estimar a distribuição da ingestão alimentar habitual em grupos. In: Fisberg RM, Slater B, Marchioni DML, Martini LA, organizadores. Inquéritos alimentares: métodos e bases científicos. Barueri: Editora Manole; 2005. p. 314-22.

34. Willett WC, Stampfer MJ. Total energy intake: implications for epidemiologic analyses. Am J Epidemiol 1986; 124:17-27.

35. Agricultural Research Service, United State Department of Agriculture. National nutrient database for standard reference, release 20. Washington DC: United States Department of Agriculture; 2007.

36. Núcleo de Estudos e Pesquisa em Alimentação. Tabela brasileira de composição de alimentos - TACO. Versão 2. 2a Ed. Campinas: Universidade Estadual de Campinas; 2006.

37. Chen XK, Wen SW, Fleming N, Demissie K, Rhoads GG, Walker M. Teenage pregnancy and adverse birth outcomes: a large population based retrospective cohort study. Int J Epidemiol 2007; 36:368-73.

38. Joseph KS, Allen AC, Dodds L, Turner LA, Scott H, Liston R. The perinatal effects of delayed childbearing. Obstet Gynecol 2005; 105:1410-8.

39. Abenhaim HA, Kinch RA, Morin L, Benjamin A, Usher R. Effect of prepregnancy body mass index categories on obstetrical and neonatal outcomes. Arch Gynecol Obstet 2007; 275:39-43.

40. Hillier TA, Pedula KL, Schmidt MM, Mullen JA, Charles MA, Pettitt DJ. Childhood obesity and metabolic imprinting: the ongoing effects of maternal hyperglycemia. Diabetes Care 2007; 30:2287-92.

41. Bellisle F. The doubly labeled water methods and food intake surveys: a confrontation. Rev Nutr PUCCAMP 2001; 14:125-33.

42. Prentice AM, Black AE, Coward WA, Cole TJ. Energy expenditure in overweight and obese adults in affluent societies: an analysis of 319 doubly-labelled water measurements. Eur J Clin Nutr 1996; 50:93-7.

43. Prentice AM, Black AE, Coward WA, Davies HL, Goldberg GR, Murgatroyd PR, et al. High levels of energy expenditure in obese women. Br Med J (Clin Res Ed) 1986; 292:983-7.

44. Fisberg RM, Slater B, Marchioni DML, Martini LA. Métodos de inquéritos alimentares. In: Fisberg RM, Slater B, Marchioni DML, Martini LA, organizadores. Inquéritos alimentares: métodos e bases científicos. Barueri: Editora Manole; 2005. p. 1-31

45. Pereira RA, Sichieri R. Métodos de avaliação do consumo de alimentos. In: Kac G, Sichieri R, Gigante DP, organizadores. Epidemiologia nutricional. Rio de Janeiro: Editora Fiocruz/Editora Atheneu; 2007. p. 181-200.

Recebido em 07/Mai/2010

Versão final reapresentada em 14/Jul/2010

Aprovado em 02/Ago/2010 